\title{
Childhood Tuberculosis Challenges and Way Forward
}

\author{
Soumya Swaminathan • Sushil Kumar Kabra
}

Received: 3 December 2010 /Accepted: 8 December 2010/Published online: 26 January 2011

(C) Dr. K C Chaudhuri Foundation 2011

Despite the increased global attention, funding and achievements in the control of tuberculosis over the past decade, children have been largely ignored and have not benefited from the advances in diagnosis and treatment. Children younger than 15 years contribute $15-20 \%$ of the global TB disease burden, although accurate data are scarce due to challenges in case ascertainment and lack of surveillance in this age group [1]. Tuberculosis transmission to children not only indicates inadequate control in the community, but creates a pool of latently infected individuals who form the fountainhead of adult disease at some time in the future. Elimination of TB by 2050, a goal of the worldwide TB scientific community requires attention to diagnosis, treatment and prevention of TB in adults and children simultaneously, though the approaches would need to be quite different [2]. Further, young children are at high risk for disease progression and high mortality as well as longterm sequelae, if the disease is not diagnosed and treated appropriately. The needs for research are substantial and it is hoped that the research community and funders will pay increasing attention to these priorities. New diagnostics including liquid culture methods, the MODS assay, nucleic

\section{S. Swaminathan}

Neglected Priorities Research, UNDP/UNICEF/World Bank/

WHO Special Programme for Research

and Training in Tropical Diseases,

Geneva, Switzerland

\section{S. K. Kabra $(\bowtie)$}

Department of Pediatrics, AIIMS,

New Delhi 110029, India

e-mail:skkabra@live.in acid amplification techniques, line probe assays and other molecular techniques need to be tested and validated on a number of different clinical specimens that may be obtained from children. Randomized trials and high quality pharmacokinetic and observational studies are required to evaluate the efficacy and safety of currently recommended dosages as well as length of anti-TB treatment for the different forms of pulmonary and extra-pulmonary TB [3]. As several new drugs enter the pipeline for the first time in decades, studies in children need to be urgently initiated if they are not to be excluded from the benefits of these advances. Surveys to estimate the burden of TB and prevalence of drug resistance should include children in an effort to generate baseline data and monitor progress of control efforts. While trials to determine the best regimen for prevention among contacts of multi-drug resistant $\mathrm{TB}$ are needed, implementation research addressing barriers to rapid scale up of contact tracing and chemoprophylaxis should take priority for TB control programs.

Indian journal of pediatrics (IJP) publishes updated reviews on clinical problems faced by pediatricians in daily clinical practice. A symposium on tuberculosis addressing the state of the art on various aspects is appearing in two parts. The articles are written by experts in the field and geared towards practicing pediatricians while highlighting gaps in knowledge. In this issue four articles on various aspects of childhood tuberculosis are included. In recent years with analysis of epidemiological aspects of childhood tuberculosis, there is better understanding of natural history of child tuberculosis. An article by Marais et al [4] discusses the epidemiology and natural history of tuberculosis. The changing trends in clinical manifestations, diagnostic approach and 
treatment is described in some detail in article by Mukherjee et al [5]. Diagnosis of childhood tuberculosis is based on indirect evidence, as isolation of Mycobacterium tuberculosis is difficult. In the absence of a gold standard, there is both under and over diagnosis of tuberculosis. In this issue, two related articles discuss the available clinical algorithms for diagnosis [6] and optimal utilization of diagnostic tests in day to day clinical practice [7].

In the second part of this symposium, articles related to pharmacokinetics of antituberculosis drugs, treatment of tuberculosis, newer diagnostic tests, drug resistant tuberculosis, management of latent tuberculosis will be published. We hope that this symposium will improve understanding and management of childhood tuberculosis.

\section{References}

1. Marais BJ, Gupta A, Starke JR, El Sony A. Tuberculosis in women and children. Lancet. 2010;6-8.

2. Lonroth K, Castro KG, Chakaya JM, et al. Tuberculosis control and elimination 2010-50: cure, care and social development. Lancet. 2010; 13-28.

3. World Health Organization. Rapid advice. Treatment of tuberculosis in children. Geneva: WHO; 2010.

4. Marais BJ. Childhood tuberculosis: epidemiology and natural history of disease. Indian J Pediatr. 2011;78. doi:10.1007/s12098-010-0353-1.

5. Mukherjee A, Lodha R, Kabra SK. Changing Trends in Childhood Tuberculosis. Indian J Pediatr. 2011;78. doi:10.1007/s12098-0100298-4.

6. Graham SM. The use of diagnostic systems for tuberculosis in children Indian J Pediatr. 2011;78. doi:10.1007/s12098-010-0307-7.

7. Amdekar YK. How to optimize current (available) diagnostic tests. Indian J Pediatr 2011;78. doi:10.1007/s12098-010-0286-8. 